\title{
Blood Pressure in Acute Ischemic Stroke
}

\author{
Michael McManus \\ David S Liebeskind \\ Neurovascular Imaging \\ Research Core \& \\ UCLA Stroke Center, \\ University of California, \\ Los Angeles, CA, USA
}

Hypertension is present in up to $84 \%$ of patients presenting with acute stroke, and a smaller proportion of patients have blood pressures that are below typical values in the context of cerebral ischemia. Outcomes are generally worse in those who present with either low or severely elevated blood pressure. Several studies have provided valuable information about malignant trends in blood pressure during the transition from the acute to the subacute phase of stroke. It is not uncommon for practitioners in clinical practice to identify what appear to be pressure-dependent neurologic deficits. Despite physiologic and clinical data suggesting the importance of blood pressure modulation to support cerebral blood flow to ischemic tissue, randomized controlled trials have not yielded robust evidence for this in acute ischemic stroke. We highlight previous studies involving acute-stroke patients that have defined trends in blood pressure and that have evaluated the safety and efficacy of blood-pressure modulation in acute ischemic stroke. This overview reports the current status of this topic from the perspective of a stroke neurologist and provides a framework for future research.

Key Words acute ischemic stroke, hypotension, hypertension, blood pressure augmentation and stroke, permissive hypertension.
Received December 1, 2014

Revised August 14, 2015

Accepted August 17, 2015

\section{Correspondence}

David S Liebeskind, MD

Neurovascular Imaging Research Core \& UCLA Department of Neurology, Neuroscience Research Building, 635 Charles E Young Drive South, Suite 225, Los Angeles,

CA 90095-7334, USA

Tel +1-310-963-5539

E-mail davidliebeskind@yahoo.com

\section{INTRODUCTION}

Hypertension (HTN) is the most common modifiable risk factor for stroke, with blood pressure (BP) reduction being associated with a reduced rate of stroke recurrence. ${ }^{1}$ However, how best to treat HTN during the acute period of ischemic stroke is less well established. In a recent study using data collected from nationwide emergency departments, $69 \%$ of presenting patients had a systolic BP (SBP) of $>139 \mathrm{~mm} \mathrm{Hg}$, while other studies have found BPs of $>150 / 90 \mathrm{~mm} \mathrm{Hg}$ in up to $84 \%$ of patients. ${ }^{2,3}$ Although the management of BP in acute ischemic stroke has been addressed in many clinical trials (Table 1), there are no definitive data showing that treatment in the hyperacute or acute setting alters clinical outcomes. ${ }^{4-7}$

Elevated BP and cerebral edema have been found to be associated with hemorrhagic transformation of cerebral infarction, but the available clinical and diagnostic data are insufficient for determining the causality of this relationship. ${ }^{8,9}$

Stroke is a complex disease with wide variations in the age at onset, etiology, comorbidities, and adequacy of collateral circulation. BP is a simple physiologic parameter that is always measured, can be modulated, and may affect the outcome in certain circumstances. The purpose of this review is to summarize the current knowledge about baseline BP, BP trends, and associated outcomes in ischemic stroke. This information will be helpful for determining where we should go from here.

\section{METHODS}

This narrative overview was carried out using PubMed and Medline to identify articles pub(a) This is an Open Access article distributed under the terms of the Creative Commons Attribution Non-Commercial License (http://creativecommons.org/licenses/by-nc/3.0) which permits unrestricted non-commercial use, distribution, and reproduction in any medium, provided the original work is properly cited. 
lished between January 1980 and September 2014 by crossreferencing the term "acute ischemic stroke" with BP, HTN, induced HTN $(\mathrm{IH})$, and antihypertensive therapy. Articles were limited to those written in English and including only human subjects with acute stroke. Randomized Phase III trials evaluating clinical outcomes in acute ischemic stroke patients were prioritized. In subtopic sections below where randomized trials were either not available or very limited, data from pilot studies, prospective cohort studies, case series, and retrospective analyses were included at the discretion of the authors. Studies limiting enrollment to hemorrhagic stroke patients were not included due the authors considering that there is insufficient physiologic overlap to extrapolate these data to ischemic infarction.

\section{CURRENT GUIDELINES AND SUGGESTED MANAGEMENT APPROACH}

According to the 2013 Guidelines of the American Heart Association (AHA) for the early management of patients with acute ischemic stroke, an ideal BP range during acute ischemic stroke has not been determined but probably depends on patient-specific factors. ${ }^{10}$ For patients eligible for intravenous (IV) tissue plasminogen activator (tPA), SBP should be lowered to $<185 \mathrm{~mm} \mathrm{Hg}$ and diastolic BP (DBP) should be lowered to $<110 \mathrm{~mm} \mathrm{Hg}$ before initiating IV thrombolytics. ${ }^{10}$ At our facility we typically administer either labetalol or hydralazine via the IV route to meet this target, with the occasional use of enalaprilat. If the BP is fluctuating near the target pressure, a nicardipine or clevidipine IV drip can be initiated to stabilize the BP at the target value. No randomized controlled studies have compared outcomes in patients requiring aggressive $\mathrm{BP}$ reduction using continuous IV antihypertensive agents to reach the target $\mathrm{BP}$ required for the administration of IV tPA versus control patients with resistant HTN considered to have a contraindication to IV tPA. In practice, SBP and DBP targets of $<180 \mathrm{~mm} \mathrm{Hg}$ and $<105 \mathrm{~mm} \mathrm{Hg}$, respectively, are practical before the tPA bolus is infused since the BP should remain below these thresholds during the infusion. AHA guidelines recommend maintaining these targets for 24 hours following IV tPA. Frequent measurement of BP every 10-15 minutes for the first 60 minutes following administration of the tPA bolus can be useful in establishing a trajectory and gathering the appropriate resources to maintain adequate control before transferring the patient to another setting. If the patient is a candidate for intra-arterial recanalization therapies, the AHA recommends SBP and DBP targets of $<180 \mathrm{~mm} \mathrm{Hg}$ and $<105$ $\mathrm{mm} \mathrm{Hg}$, respectively. In the particular instances of stroke ne- cessitating angioplasty and/or stenting of an artery suspected to have chronic flow-limiting stenosis, the target BP may be lowered further depending upon the clinician's concern about reperfusion syndrome.

In patients not receiving IV thrombolytics or intra-arterial recanalization therapies, the AHA recommends withholding antihypertensive therapies unless SBP is $>220 \mathrm{~mm}$ $\mathrm{Hg}$ or DBP is $>120 \mathrm{~mm} \mathrm{Hg}$. AHA guidelines also state that it is reasonable to lower BP by $15 \%$ during the first 24 hours after stroke onset (evidence for this recommendation is detailed in later sections). Lower BP targets are often initiated if there is evidence of end-organ damage due to elevated BP or if an elevated pressure is thought to be exacerbating a comorbid condition.

In our facility, antihypertensive medications are typically initiated 24 hours after the start of symptomatic cerebral ischemia if the patient is clinically stable or improving. These medications are chosen according to the current JNC 8 (Joint Committee on Prevention Detection, Evaluation, and Treatment of High BP) guidelines.

\section{BP TRENDS IN ACUTE STROKE}

The BP is typically at maximal at admission but decreases spontaneously thereafter during the natural course of stroke. ${ }^{11,12}$ While this temporal pattern also occurs in control patients without a diagnosis of stroke, the BPs are higher in stroke patients. ${ }^{11}$ One study found that the SBP on arrival significantly higher in those with ischemic lacunar and thromboembolic infarcts than in controls (mean, 13.2\% and 8.6\%, respectively), with similar elevations in DBP, but the differences were no longer significant at repeat measures on day 7. ${ }^{13}$ Spontaneous reductions in BP continue for several days after the event. ${ }^{3}$

\section{OBSERVATIONS OF BP IN THE ACUTE SETTING AND ASSOCIATED OUTCOMES}

There is a U-shaped relationship between outcomes and the admission $\mathrm{BP}$ in the acute setting. ${ }^{7.14} \mathrm{~A}$ retrospective analysis of data from the International Stroke Trial evaluated outcomes associated with the prerandomization BP in patients with acute ischemic stroke, and found U-shaped relationships of baseline SBP with early death, late death, and dependency. The risk of early death increased for every 10 $\mathrm{mm} \mathrm{Hg}$ increase for SBP $<150 \mathrm{~mm} \mathrm{Hg}$, and by $3.8 \%$ for every $10 \mathrm{~mm} \mathrm{Hg}$ increase for SBP $>150 \mathrm{~mm} \mathrm{Hg}$. That study also found that the rate of recurrent ischemic stroke within 14 days increased by $4.2 \%$ for every $10 \mathrm{~mm} \mathrm{Hg}$ increase in 
SBP, but no relationship between symptomatic intracranial hemorrhage and SBP was found, while low SBP was associated with severe anterior circulation stroke syndrome and death from coronary artery disease. ${ }^{7}$ Another study identified a minimum SBP of $130 \mathrm{~mm} \mathrm{Hg}$ in the U-shaped relationship. ${ }^{15}$ The 1-month and 1-year mortality rates showed relative-risk increases of $10.2 \%$ and $7.2 \%$, respectively, for every $10 \mathrm{~mm} \mathrm{Hg}$ increase for SBP $>130 \mathrm{~mm} \mathrm{Hg}$. There was a relative-risk increase in mortality of $28.2 \%$ at 1 month and $17.5 \%$ at 1 year for every $10 \mathrm{~mm} \mathrm{Hg}$ decrease below the minimum SBP, and low admission BPs were associated with heart failure and coronary artery disease..$^{15}$ In 2004 Castillo et al. ${ }^{16}$ produced similar findings of $\mathrm{U}$-shaped relationships of the admission BP with an outcome of early neurologic deterioration (END) and poor neurologic outcome, but found that the risk was lowest in those with an admission BP of around 180/100 mm Hg.

A more recent study found no J- or U-shaped relationship, instead finding that a higher pressure was associated with a lower probability of good neurologic recovery during hospitalization, a higher risk of neurologic deterioration during hospitalization, and poor functional outcome at 3 months. ${ }^{17}$ The methodology for calculating average BPs in that study did not allow for trends to be followed in the acute period, and therefore could have grouped patients with appropriate BP reductions with those who had persistent elevations over a 48-hour period. Moreover, patients with baseline impairment in the activities of daily living were excluded, and the percentages of patients with coronary artery disease or heart failure were not reported.

In patients with prior HTN, the relationship between prognosis and SBP in the acute setting has been shown to shift significantly toward higher pressures (by about $10 \mathrm{~mm} \mathrm{Hg}$ ) compared with those without prior HTN. ${ }^{14}$ This underlines the importance of considering relative rather than absolute $\mathrm{BP}$ changes when assessing acute stroke.

\section{OBSERVATIONAL STUDIES OF BP TREND VERSUS OUTCOMES}

The 2004 study by Castillo et al. ${ }^{16}$ evaluated the association between early trends in BP in 304 consecutive hemispheric acute ischemic stroke patients within 24 hours of onset. Reductions in SBP and DBP of $>20 \mathrm{~mm} \mathrm{Hg}$ during the first 24 hours were associated with a higher frequency of END, increased infarct volume, and worse outcome at 3 months.

Data from acute ischemic stroke patients treated with antihypertensive agents in accordance with 2008 European Stroke Organization guidelines showed the best outcomes in patients with acute SBP values between $110 \mathrm{~mm} \mathrm{Hg}$ and 180 $\mathrm{mm} \mathrm{Hg}$ followed by an SBP reduction over $24-48$ hours. $^{18}$ The optimal reductions in SBP were found to vary with the acute SBP: reductions of $10-20 \%$ and $>20 \%$ during the first 24-48 hours after arrival produced better outcomes in those with acute SBP values of $120-140 \mathrm{~mm} \mathrm{Hg}$ and $140-170 \mathrm{~mm}$ $\mathrm{Hg}$, respectively. However, the stroke subtype was not controlled in those subjects. High BP during the first 48 hours after ischemic stroke as well as increases in BP over the first 24-48 hours have been repeatedly associated with worse outcomes, but there are no data supporting elevated BP as a causative mechanism for such outcomes. ${ }^{17-19}$

\section{USE OF ANTIHYPERTENSIVE AGENTS AND ASSOCIATED OUTCOMES IN THE ACUTE SETTING}

The known benefits of angiotensin-converting enzyme inhibitors (ACE-I) in myocardial infarction have led to investigations of its potential benefit in acute ischemic stroke. The Evaluation of Acute Candesartan Cilexetil Therapy in Stroke Survivors (ACCESS) study compared the effects of an SBP reduction of $10-15 \%$ within 24 hours of randomization in patients with acute stroke with placebo. Functional dependency did not differ between these two groups at 3 months, and there was no group difference in cerebrovascular events at 12 months. ${ }^{20}$ There was a reduction in the secondary end points of 12-month mortality ( 5 events vs. 12 events, $p=0.07$ ) and vascular events (17 events vs. 31 events, $p=0.026)$ in favor of the treatment group..$^{20}$ In that study the BPs did not differ between the treatment and control groups on admission, during the 7 day placebo-controlled period, or during the 12-month follow-up period. ${ }^{20}$ Unlike subsequent studies, the ACCESS study population only included patients with severe BP elevations. That study did not address the effect of $\mathrm{BP}$ reduction in the acute setting, but it did obtain some interesting data on the potential long-term benefits of ACE-I administered during the time window of 24-36 hours after acute ischemic stroke via mechanisms other than $\mathrm{BP}$ reduction.

A substudy of the Prevention Regimen for Effectively Avoiding Second Stroke (ProFESS) ${ }^{21}$ trial revisited potential benefits of angiotensin-mediated antihypertensive agents using telmisartan in acute ischemic stroke patients. ${ }^{22}$ At 7 days the BPs were significantly lower in the treatment group (mean reductions in SBP and DBP of 6.1 and $3.2 \mathrm{~mm} \mathrm{Hg}$, respectively; $p<0.0001)$. However, no differences were found in the combined 30-day death or dependency rate, or in the 90-day death rate or stroke recurrence. Most patients were recruited during the third day after stroke onset with a mean National Institutes of Health Stroke Scale (NIHSS) score of 
3. The population in that ProFESS substudy had SBPs of 121-180 mm Hg, which are markedly lower than for those included in the ACCESS trial.

A similar study, the Scandinavian Candesartan Acute Stroke Trial (SCAST), randomized 2,029 patients with acute ischemic or hemorrhagic stroke to therapy with candesar$\tan$ (which is an angiotensin-receptor blocker) or placebo within 30 hours of onset. ${ }^{23}$ On day 7 the BP was significantly lower in the candesartan group, but the 6-month composite end point of vascular death, myocardial infarction, or stroke did not differ between the treatment and placebo groups. A post-hoc analysis was performed to evaluate the effect of BP lowering in patients with carotid artery stenosis, which revealed that progressive stroke was more frequent in patients with carotid stenosis treated with candesartan: $11.5 \%$ and $4 \%$ in the treatment and control groups, respectively. ${ }^{24}$ Furthermore, the risk increased with the severity of stenosis ( $p$ for linear trend=004). ${ }^{24}$

The CHHIPS (Controlling HTN and Hypotension Immediately Post-Stroke) trial compared the effects of lisinopril, labetalol, and placebo on the 2-week death or dependency rate when administered between 5 and 36 hours from the onset of ischemic or hemorrhagic stroke. The decrease in SBP was significantly greater in the combined active treatment group than in the placebo group during the first 24 hours [difference: mean $=10 \mathrm{~mm} \mathrm{Hg}, 95 \%$ confidence inter$\operatorname{val}(\mathrm{CI})=3-17 \mathrm{~mm} \mathrm{Hg} ; p=0.004]$. However, there was no difference in the primary end point of the 2-week death or dependency rate within treatment groups or between the treatment and placebo groups. ${ }^{12}$

The evidence supporting the safety of BP reduction in acute stroke ${ }^{12,20}$ and the many stroke patients who present with symptoms after using prescribed antihypertensive agents prompted interest in evaluating the effect of continuation of home antihypertensive agents in acute-stroke patients. Acute-stroke patients were randomized within 48 hours of onset to discontinue all antihypertensive therapies or to continue their home regimen for a period of 2 weeks in the Continue Or Stop post-Stroke Antihypertensives Collaborative Study. ${ }^{25}$ The baseline BP was not significantly different in the treatment and control groups, but no further BP comparisons were provided in the acute setting. At 2 weeks the $\mathrm{BP}$ differed significantly between the treatment and nontreatment groups (difference in SBP/DBP: mean $=13 / 8 \mathrm{~mm}$ $\mathrm{Hg}$, 95\% CI=10-17/6-10 mm Hg; $p \leq 0.0001$ ), whereas there was no group difference in the primary outcome of risk of death or dependency. ${ }^{25}$ The study population was somewhat restricted by dysphasic patients being excluded due to the need to continue home oral medications. Furthermore, in order to enhance enrollment, eligibility from the time of symptom onset was increased from 24 hours to 48 hours and patients with a prestroke score on the modified Rankin Scale (mRS) of $\leq 3$ were included. ${ }^{25}$

More recently, the CATIS (China Antihypertensive Trial in Acute Ischemic Stroke) randomized clinical trial focused on acute ischemic stroke and the effects of immediate BP reduction on death and major disability using a defined SBP reduction target of 10-25\% within 24 hours of randomization and a BP of $<140 / 90 \mathrm{~mm} \mathrm{Hg}$ within 7 days versus no antihypertensive therapy. ${ }^{26}$ Antihypertensive agents were chosen for that trial based on a predefined algorithm and included IV ACE-I, calcium-channel blockers, and diuretics. Significant reductions in BP between the treatment and control groups were achieved at both 24 hours and 14 days after randomization. The primary combined end point of the death or dependency rate at 14 days or hospital discharge, and the secondary end point of the mRS score at 3 months did not differ between the treatment and control groups. ${ }^{26}$ Interestingly, there was a significant reduction in the primary end point in the treatment group when antihypertensive agents were started $\geq 24$ hours after symptom onset.

\section{OBSERVATIONAL STUDIES OF BP AFTER IV THROMBOLYSIS}

While IV thrombolysis is applied to a significant proportion of acute ischemic stroke patients, investigations into the association between HTN and outcomes are restricted in this population by guidelines excluding the use of IV thrombolytics in patients with severe HTN. It is important to note that the upper limits of BP $(185 / 110 \mathrm{~mm} \mathrm{Hg})$ derive from pilot data obtained before the landmark NINDS (National Institute of Neurological Disorders and Stroke) tPA trial and data from the use of tPA in myocardial infarction. In 2007, Lansberg et al. ${ }^{27}$ reviewed studies incorporating multivariate analysis to identify independent risk factors of symptomatic intracerebral hemorrhage (sICH) associated with thrombolytic therapy for stroke. Eight of the studies evaluated HTN as a variable, and only one study (using streptokinase) found a significant association between $\mathrm{BP}$ and $\mathrm{sICH}$. Three subsequent studies have evaluated $\mathrm{HTN}$ as a variable in sICH after IV thrombolysis.

The SITS-ISTR (Safe Implementation of Thrombolysis in Stroke-International Stroke Thrombolysis Register) ${ }^{28}$ database was used in a retrospective analysis of the relationships between $\mathrm{BP}$ and the development of type $2 \mathrm{sICH}$, mortality, and independence at 3 months. ${ }^{8}$ The BP was recorded at baseline and 2 and 24 hours after administering IV thrombolytics. As a continuous variable, a high BP was associated with a worse outcome $(p=0.001)$, while as a categorical vari- 
able the $\mathrm{BP}$ was linearly associated with sICH and a U-shaped association with mortality and independence. An SBP of 141-150 $\mathrm{mm} \mathrm{Hg}$ was associated with the most favorable outcomes. ${ }^{8}$ However, indications, methods, and rates of BP modulation were not reported, and there was no analysis of the effect of large-artery occlusion or if recanalization was achieved. In clinical practice, the reasoning behind initiating or withholding antihypertensive agents is often related to the degree of suspicion for ongoing or progressive cerebral ischemia.

Retrospective analysis of the Echoplanar Imaging Thrombolytic Evaluation Trial (EPITHET) data examined the risk of hemorrhage after IV thrombolysis with IPA in acute ischemic stroke within the time window of 3-6 hours poststroke. ${ }^{29}$ Diffusion restriction volumes on MRI were not used to determine eligibility for thrombolytic treatment, and these volumes were significantly larger in those with hemorrhage than in those without hemorrhage. However, the baseline SBP did not differ between the hemorrhage and nonhemorrhage groups, whereas the weighted average SBP during the 24 hours following treatment was higher in those with hemorrhage [159.4 $\pm 18.8 \mathrm{~mL}($ mean $\pm \mathrm{SD}), p<0.011$ ] than in those without hemorrhage $(143.1 \pm 20.0 \mathrm{~mL}$ ). Logistic regression analysis indicated that hemorrhage was predicted not only by the 24 -hour weighted average SBP but also by the diffusion-weighted imaging lesion volume and the presence of atrial fibrillation. ${ }^{29}$

A more recent, single-center Danish study evaluated the relationship between BP and outcomes after IV tPA using the 4.5 -hour poststroke time window. ${ }^{30}$ The following four quartiles based on the admission SBP were defined: $<143 \mathrm{~mm}$ $\mathrm{Hg}, 143-163 \mathrm{~mm} \mathrm{Hg}, 164-181.5 \mathrm{~mm} \mathrm{Hg}$ and $>181.5 \mathrm{~mm} \mathrm{Hg}$. There was a trend toward the short- and long-term outcomes being worse in quartiles with the lowest and highest admission SBPs than in the quartile with an admission SBP of 143$163 \mathrm{~mm} \mathrm{Hg}$. However, the canalization status, perfusion data, and incidence of hemorrhage were not reported.

\section{BP AUGMENTATION IN ISCHEMIC STROKE}

Animal models have shown improved cerebral blood flow $(\mathrm{CBF})$ and reduced cerebral edema in the infarction territory after administering phenylephrine following a focal infarction. ${ }^{31,32}$ Although BP augmentation is often used in the setting of ischemic stroke, no large randomized control studies have assessed the safety and efficacy of this treatment. The sections above have established that hypotension following ischemic cerebral infarction is associated with a poor outcome. A pilot study performed in 2001 aimed to deter- mine the safety and feasibility of phenylephrine-IH in acute ischemic stroke. ${ }^{33}$ That group also intended to identify the subset of patients with neurologic deficits that were responsive to BP augmentation. All patients were outside the poststroke time window for IV thrombolysis, and most of them were determined to have ischemic stroke of embolic etiology affecting the middle cerebral artery (MCA) territory. Phenylephrine was used to increase the SBP to a target of $160 \mathrm{~mm}$ $\mathrm{Hg}$ or by $20 \%$ relative to the admission BP, and without exceeding $200 \mathrm{~mm} \mathrm{Hg} .{ }^{33}$ Thirteen patients were enrolled within 12 hours of stroke onset, of which 7 improved by 2 points on the NIHSS after the target BP was obtained, and 4 of these responders had a proximal MCA occlusion. A BP threshold was found in six patients, and no systemic or neurologic complications were reported.

Koenig et al. ${ }^{34}$ retrospectively evaluated adverse events and outcomes associated with $\mathrm{IH}$ in 100 serial ischemic stroke patients undergoing perfusion MRI. Patients receiving IV thrombolytics or endovascular intervention were excluded, and IH was initiated at the discretion of the treatment team and with the intention to increase BP by $10^{-}$ $20 \%$ above baseline. The prevalence of severe large-vessel disease was significantly higher in the IH group (54\%) than in the standard-therapy group (11\%), as was the frequency of $>70 \%$ stenosis of a major intracranial or extracranial artery. Diffusion-perfusion mismatch was greater in the $\mathrm{IH}$ group, and IH therapy was initiated at $6.5 \pm 8.0$ hours from the time of MRI-substantially less time than the mean treatment times achieved in the main trials involving the use of antihypertensive therapy in acute stroke. There was no significant difference in rate of adverse events in the $\mathrm{IH}$ and standard-therapy groups, and there were no deaths among the study population. Only one of the patients in the IH group who achieved a target mean arterial pressure (MAP) increase experienced an adverse event, which was sICH after the MAP was elevated by $35 \%$ using IV pressors.

A randomized trial by Hillis et al. ${ }^{35}$ evaluated the effect of IH in 17 consecutive ischemic stroke patients up to 7 days from symptom onset with large diffusion-perfusion mismatch. The subjects were randomly assigned to IH using phenylephrine or conventional management if $>20 \%$ diffusion-perfusion mismatch was present. IV phenylephrine was titrated to achieve a $10-20 \%$ increase in MAP over 1-8 hours, and this target was maintained for at least 24 hours. All patients had severe stenosis or occlusion of the MCA or distal internal carotid artery. Each patient in the intervention group showed some degree of improvement on the NIHSS and a cognitive battery after the treatment target was reached. On day 3 the NIHSS score had improved by $4.2 \pm 1.0$ in the intervention group and $1.2 \pm 3.0$ in the control group. 


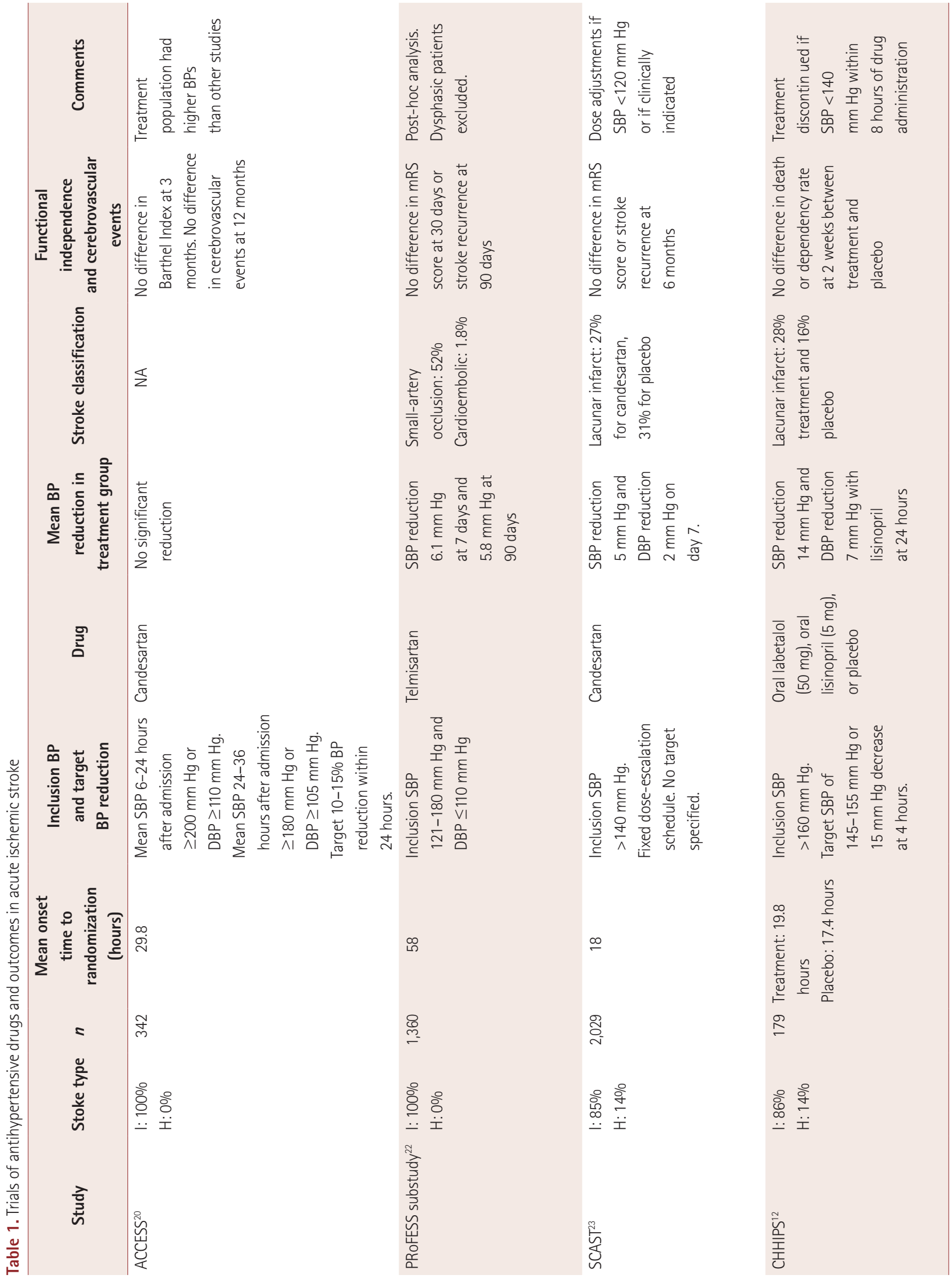




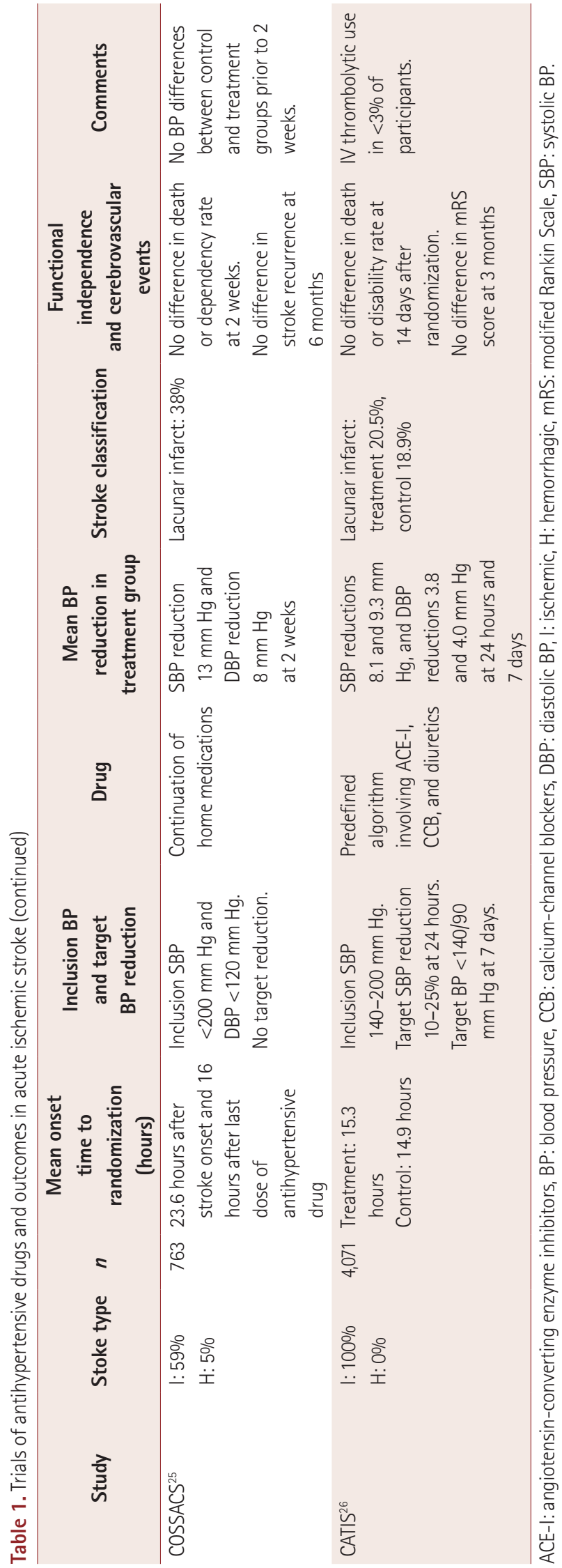

The NIHSS scores did not differ significantly between the groups on day 1 , but on day 3 they were significantly lower in the treatment group $(5.6 \pm 6.0$ vs. $9.7 \pm 8.0$, median $=3$ vs. $11 ; p<0.02)$. Treated patients also had significantly better NIHSS scores compared with control patients in the longterm follow-up ( $2.8 \pm 4.0$ vs. $9.7 \pm 8.0$, median $=2$ vs. 12.5 ; $\mathrm{z}=1.8, p<0.04)$. There was no significant reduction in the volume of hypoperfused tissue in perfusion-weighted imaging between pretreatment and an immediate follow-up. No subjects in the treatment group developed cardiac ischemia or hemorrhagic transformation of infarction.

The Safety and Efficacy of Therapeutic INduced HYPERTENSION in acute non-cardioembolic ischemic stroke (SETIN-HYPERTENSION) trial is a multicenter, randomized, open-label, prospective, phase-III trial that aims to determine the safety and efficacy of IH using phenylephrine in patients with noncardioembolic ischemic stroke. That study has the potential to yield valuable data for this topic, and subjects are currently being enrolled.

There is a paucity of data supporting the safety and potential efficacy of BP augmentation in hypotensive patients with large-vessel occlusions. Future studies should therefore evaluate the potential benefits of BP augmentation in patients with acute cardioembolic stroke who are not eligible for recanalization therapies or who have received IV thrombolytics and have persistent occlusions that cannot be addressed by intra-arterial therapies.

\section{DISCUSSION}

The definition of "permissive HTN" is unique to the individual under particular vascular conditions and during a particular phase in the course of an ischemic event. In effect, this indicates that the cerebral perfusion is dependent on the systemic pressure. CBF remains independent of MAP under normal physiologic conditions. Primate models of cerebral ischemia have demonstrated that the regional CBF in electrically silent but not-yet-infarcted tissue is correlated with MAP. ${ }^{36}$ In human subjects, the regional CBF in the ischemic penumbra has also been shown to be pressure dependent; ${ }^{4}$ this is related to the concern over early antihypertensive therapy in patients with ischemic but not-yet-infarcted tissue. The goal of permissive HTN is to optimize blood flow to the ischemic penumbra until IV thrombolytics can be administered, and intra-arterial recanalization therapies can be employed or optimization of the collateral vasculature can occur.

While multiple independent studies have found a Ushaped association between admission BP and outcomes, ${ }^{7,14}$ these data should be interpreted with caution. Although sev- 
eral studies have found the SBP for moderate HTN to be associated with the best outcomes, the BP alone should not only be seen as a potential marker for the outcome but also as a reflection of the underlying physiology in the individual patient. Persistent severe elevations in BP and further increases in BP during the subacute phase have been associated with worse outcomes, but this does not reliably imply a causative relationship. ${ }^{16,18}$ Instead of aiming toward unidirectional protocols for BP adjustment as a means to affect outcome, both clinicians and researchers alike need to first consider why the BP deviates beyond expected values in a clinical examination and use appropriate imaging modalities to help determine the perfusion adequacy.

Stroke is a complex disease. As recently demonstrated in positive trials of mechanical thrombectomy for the occlusion of large vessels in the anterior circulation, appropriate patient selection is paramount for achieving the potential benefits of interventions in the acute poststroke window. Most studies of antihypertensive agents have not evaluated the effect of small- versus large-vessel occlusions and have not evaluated the status of the collateral vasculature. Data from the BP-augmentation literature and the SCAST carotid substudy have shown that large-artery occlusions are more likely to result in derangements in $\mathrm{CBF}$ that are more sensitive to systemic BP. ${ }^{24,34,35}$ Future research should separately consider large- and small-vessel occlusions and attempt to incorporate the status of the collateral circulation in the analyses.

It has been established that cerebral infarction and intraparenchymal hemorrhage increase BP, but it has not been established that increased BP in the acute setting causes malignant edema. HTN is presumed to increase the permeability of the blood-brain barrier, but there is no established association with severe cerebral edema after stroke. ${ }^{37,38}$ Previous studies of the potential association between HTN and hemorrhagic conversion of infarction have produced conflicting results and definitions of hemorrhage. ${ }^{7,29,39}$ However, hemorrhage has been a consistent finding in the context of large pretreatment infarct volumes, and the associations between risk of hemorrhage and elevated BP appear to be more consistent in study populations receiving IV thrombolytics. ${ }^{8,29,39}$

The present evidence supports that a failure to circumvent progression of cerebral ischemia leads to a larger infarct volume and higher risks of malignant cerebral edema and symptomatic hemorrhagic conversion of the infarction. Although persistent and severe BP elevations probably have a role in the development of these phenomena, the focus should remain on strategies that can ameliorate cerebral ischemia.

Many randomized control studies of antihypertensive therapies in acute stroke have enrolled both ischemic and hemorrhagic stroke patients. Although large randomized control trials are still in progress, multiple studies have suggested that an elevated BP increases the risk of hematoma enlargement and that aggressive reduction of the BP reduces the risk of neurologic deterioration in spontaneous acute intracerebral hemorrhage. ${ }^{40,41}$ We also have obtained some evidence that BP elevation in a subset of patients with largevessel occlusions improves cerebral perfusion and outcomes. ${ }^{35}$ It may not be valid to liken ischemia caused by cerebrovascular occlusions to that caused by cerebrovascular rupture due to the inherent difference between the hemodynamic challenges that need to be addressed to support CBF through the site of injury while avoiding hemorrhagic complications.

Trials of antihypertensive agents that have focused on acute ischemic stroke have not identified significant differ-

Table 2. Observational studies of BP and outcomes after IV thrombolysis

\begin{tabular}{|c|c|c|c|}
\hline Study & Treatment & $n$ & Outcomes \\
\hline $\begin{array}{l}\text { SITS-ISTR } \\
\text { (retrospective analysis) }^{8}\end{array}$ & IV tPA within 3 hours of symptom onset & 11,080 & $\begin{array}{l}\text { High BP (continuous variable) associated with worse } \\
\text { outcome. } \\
\text { Linear association between BP and sICH, and U-shaped } \\
\text { association with mortality and independence }\end{array}$ \\
\hline 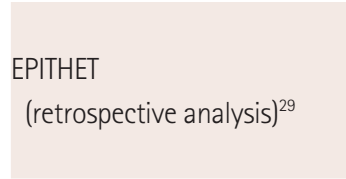 & $\begin{array}{l}\text { Early ischemic changes on CT in <one-third of } \\
\text { the MCA territory. IV tPA vs. placebo 3-6 hours } \\
\text { after symptom onset }\end{array}$ & 97 & $\begin{array}{l}\text { Average SBP } 24 \text { hours after } \mathrm{PA} \text { higher in } \mathrm{PH} \text { group } \\
(159.4 \text { vs. } 143.1 \mathrm{~mm} \mathrm{Hg}, \mathrm{p}<0.011) \\
\text { No difference in baseline BP between } \mathrm{PH} \text { and no-PH } \\
\text { group }\end{array}$ \\
\hline $\begin{array}{l}\text { Bentsen et al. } 2013 \\
{\text { (prospective cohort study) }{ }^{30}}^{\text {pos }}\end{array}$ & IV tPA within 4.5 hours of symptom onset & 265 & $\begin{array}{l}\text { Trend toward negative influence in NIHSS score change } \\
\text { at } 24 \text { hours and mRS score at } 3 \text { months in those with } \\
\text { low and high BP. Best outcomes for SBP range of } \\
143-163 \mathrm{~mm} \mathrm{Hg}\end{array}$ \\
\hline
\end{tabular}

BP: blood pressure, IV: intravenous, MCA: middle cerebral artery, mRS: modified Rankin Scale, NIHSS: National Institutes of Health Stroke Scale, PH: parenchymal hemorrhage, SBP: systolic BP, sICH: symptomatic intracerebral hemorrhage, tPA: tissue plasminogen activator. 
ences in outcomes between the treatment and control groups. Most studies have found very mild reductions in BP $(<10 \%$ from baseline values), and all randomized control studies have set an enrollment window of $>24$ hours from symptom onset. Although retrospective analyses of previous data will probably continue, only a small fraction of the patients included in large randomized trials were randomized at $<24$ hours from symptom onset. Neurologic deterioration in acute ischemic stroke typically occurs within 24 hours of the onset, and interventional therapies are typically employed within 6 hours of symptom onset. Attempts at pharmacologic modulation of BP to support the ischemic penumbra should be applied earlier, and future studies should consider the most practical and effective time windows. At this time there is a paucity of data on the impacts of pharmacologic elevation and reduction of BP in the hyperacute and early acute settings.

The information we have obtained from trials of BP modulation in acute ischemic stroke has been extremely valuable in helping to identify the relative safety of modest adjustments in BP. We have developed clues to help identify subgroups that may benefit the most from aggressive BP modulation, depending upon patient-specific factors such as location of the occlusion, status of the collateral circulation, BP trends within the individual, and other medical comorbidities. The continuing expansion of electronic medical records is improving the ability to obtain useful information about the premorbid BP so as to better assist early management decisions. The potential benefits of serial perfusion weighted imaging in the acute setting in providing feedback about the status of blood flow in the ischemic penumbra need to be explored further. There is also a lack of data on the effects of BP modulation following endovascular recanalization. Recent studies demonstrating the benefits of endovascular therapies in acute ischemic stroke have the potential to increase the number of patients available to participate in such studies and contribute to our ability to further individualize care in this complex disease.

\section{Conflicts of Interest}

The authors have no financial conflicts of interest.

\section{REFERENCES}

1. Arima H, Chalmers J, Woodward M, Anderson C, Rodgers A, Davis $\mathrm{S}$, et al. Lower target blood pressures are safe and effective for the prevention of recurrent stroke: the PROGRESS trial. J Hypertens 2006;24:1201-1208.

2. Qureshi AI, Ezzeddine MA, Nasar A, Suri MF, Kirmani JF, Hussein HM, et al. Prevalence of elevated blood pressure in 563,704 adult patients with stroke presenting to the ED in the United States. Am J Emerg Med 2007;25:32-38.

3. Wallace JD, Levy LL. Blood pressure after stroke. JAMA 1981;246:
2177-2180.

4. Olsen TS, Bruhn P, Oberg RG. Cortical hypoperfusion as a possible cause of 'subcortical aphasia'. Brain 1986;109(Pt 3):393-410.

5. Lee JM, Zhai G, Liu Q, Gonzales ER, Yin K, Yan P, et al. Vascular permeability precedes spontaneous intracerebral hemorrhage in strokeprone spontaneously hypertensive rats. Stroke 2007;38:3289-3291.

6. Ueno M, Sakamoto H, Tomimoto H, Akiguchi I, Onodera M, Huang $\mathrm{CL}$, et al. Blood-brain barrier is impaired in the hippocampus of young adult spontaneously hypertensive rats. Acta Neuropathol 2004; 107:532-538.

7. Leonardi-Bee J, Bath PM, Phillips SJ, Sandercock PA; IST Collaborative Group. Blood pressure and clinical outcomes in the International Stroke Trial. Stroke 2002;33:1315-1320.

8. Ahmed N, Wahlgren N, Brainin M, Castillo J, Ford GA, Kaste M, et al. Relationship of blood pressure, antihypertensive therapy, and outcome in ischemic stroke treated with intravenous thrombolysis: retrospective analysis from Safe Implementation of Thrombolysis in Stroke-International Stroke Thrombolysis Register (SITS-ISTR). Stroke 2009;40:2442-2449.

9. Mazya M, Egido JA, Ford GA, Lees KR, Mikulik R, Toni D, et al. Predicting the risk of symptomatic intracerebral hemorrhage in ischemic stroke treated with intravenous alteplase: safe Implementation of Treatments in Stroke (SITS) symptomatic intracerebral hemorrhage risk score. Stroke 2012;43:1524-1531.

10. Jauch EC, Saver JL, Adams HP Jr, Bruno A, Connors JJ, Demaerschalk BM, et al. Guidelines for the early management of patients with acute ischemic stroke: a guideline for healthcare professionals from the American Heart Association/American Stroke Association. Stroke 2013;44:870-947.

11. Britton M, Carlsson A, de Faire U. Blood pressure course in patients with acute stroke and matched controls. Stroke 1986;17:861-864.

12. Potter JF, Robinson TG, Ford GA, Mistri A, James M, Chernova J, et al. Controlling hypertension and hypotension immediately poststroke (CHHIPS): a randomised, placebo-controlled, double-blind pilot trial. Lancet Neurol 2009;8:48-56.

13. Morfis L, Schwartz RS, Poulos R, Howes LG. Blood pressure changes in acute cerebral infarction and hemorrhage. Stroke 1997;28:1401-1405.

14. Okumura K, Ohya Y, Maehara A, Wakugami K, Iseki K, Takishita S. Effects of blood pressure levels on case fatality after acute stroke. $J$ Hypertens 2005;23:1217-1223.

15. Vemmos KN, Tsivgoulis G, Spengos K, Zakopoulos N, Synetos A, Manios E, et al. U-shaped relationship between mortality and admission blood pressure in patients with acute stroke. J Intern Med 2004; 255:257-265.

16. Castillo J, Leira R, García MM, Serena J, Blanco M, Dávalos A. Blood pressure decrease during the acute phase of ischemic stroke is associated with brain injury and poor stroke outcome. Stroke 2004;35: 520-526.

17. Ishitsuka K, Kamouchi M, Hata J, Fukuda K, Matsuo R, Kuroda J, et al. High blood pressure after acute ischemic stroke is associated with poor clinical outcomes: Fukuoka Stroke Registry. Hypertension 2014; 63:54-60.

18. Ntaios G, Lambrou D, Michel P. Blood pressure change and outcome in acute ischemic stroke: the impact of baseline values, previous hypertensive disease and previous antihypertensive treatment. J Hypertens 2011;29:1583-1589.

19. Willmot M, Leonardi-Bee J, Bath PM. High blood pressure in acute stroke and subsequent outcome: a systematic review. Hypertension 2004;43:18-24.

20. Schrader J, Lüders S, Kulschewski A, Berger J, Zidek W, Treib J, et al. The ACCESS Study: evaluation of Acute Candesartan Cilexetil Therapy in Stroke Survivors. Stroke 2003;34:1699-1703.

21. Diener HC, Sacco RL, Yusuf S, Cotton D, Ounpuu S, Lawton WA, et al. Effects of aspirin plus extended-release dipyridamole versus clopidogrel and telmisartan on disability and cognitive function after re- 
current stroke in patients with ischaemic stroke in the Prevention Regimen for Effectively Avoiding Second Strokes (PRoFESS) trial: a double-blind, active and placebo-controlled study. Lancet Neurol 2008;7:875-884.

22. Bath PM, Martin RH, Palesch Y, Cotton D, Yusuf S, Sacco R, et al. Effect of telmisartan on functional outcome, recurrence, and blood pressure in patients with acute mild ischemic stroke: a PRoFESS subgroup analysis. Stroke 2009;40:3541-3546.

23. Sandset EC, Bath PM, Boysen G, Jatuzis D, Kõrv J, Lüders S, et al. The angiotensin-receptor blocker candesartan for treatment of acute stroke (SCAST): a randomised, placebo-controlled, double-blind trial. Lancet 2011;377:741-750.

24. Jusufovic M, Sandset EC, Bath PM, Karlson BW, Berge E; Scandinavian Candesartan Acute Stroke Trial Study Group. Effects of blood pressure lowering in patients with acute ischemic stroke and carotid artery stenosis. Int J Stroke 2015;10:354-359.

25. Robinson TG, Potter JF, Ford GA, Bulpitt CJ, Chernova J, Jagger C, et al. Effects of antihypertensive treatment after acute stroke in the Continue or Stop Post-Stroke Antihypertensives Collaborative Study (COSSACS): a prospective, randomised, open, blinded-endpoint trial. Lancet Neurol 2010;9:767-775.

26. He J, Zhang Y, Xu T, Zhao Q, Wang D, Chen CS, et al. Effects of immediate blood pressure reduction on death and major disability in patients with acute ischemic stroke: the CATIS randomized clinical trial. JAMA 2014;311:479-489.

27. Lansberg MG, Albers GW, Wijman CA. Symptomatic intracerebral hemorrhage following thrombolytic therapy for acute ischemic stroke: a review of the risk factors. Cerebrovasc Dis 2007;24:1-10

28. Wahlgren N, Ahmed N, Dávalos A, Ford GA, Grond M, Hacke W, et al. Thrombolysis with alteplase for acute ischaemic stroke in the Safe Implementation of Thrombolysis in Stroke-Monitoring Study (SITSMOST): an observational study. Lancet 2007;369:275-282.

29. Butcher K, Christensen S, Parsons M, De Silva DA, Ebinger M, Levi C, et al. Postthrombolysis blood pressure elevation is associated with hemorrhagic transformation. Stroke 2010;41:72-77.

30. Bentsen L, Ovesen C, Christensen AF, Christensen H. Does the admission blood pressure associate with short- and long term outcome in stroke patients treated with thrombolysis? A single centre study.
Int J Hypertens 2013;2013:610353.

31. Drummond JC, Oh YS, Cole DJ, Shapiro HM. Phenylephrine-induced hypertension reduces ischemia following middle cerebral artery occlusion in rats. Stroke 1989;20:1538-3544.

32. Drummond JC, Oh YS, Cole DJ. Does phenylephrine-induced hypertension during focal cerebral ischemia aggravate brain edema? J Neurosurg Anesthesiol 1989;1:120-121.

33. Rordorf G, Koroshetz WJ, Ezzeddine MA, Segal AZ, Buonanno FS. A pilot study of drug-induced hypertension for treatment of acute stroke. Neurology 2001;56:1210-1213.

34. Koenig MA, Geocadin RG, de Grouchy M, Glasgow J, Vimal S, Restrepo L, et al. Safety of induced hypertension therapy in patients with acute ischemic stroke. Neurocrit Care 2006;4:3-7.

35. Hillis AE, Ulatowski JA, Barker PB, Torbey M, Ziai W, Beauchamp $\mathrm{NJ}$, et al. A pilot randomized trial of induced blood pressure elevation: effects on function and focal perfusion in acute and subacute stroke. Cerebrovasc Dis 2003;16:236-246.

36. Astrup J, Symon L, Branston NM, Lassen NA. Cortical evoked potential and extracellular $\mathrm{K}+$ and $\mathrm{H}+$ at critical levels of brain ischemia. Stroke 1977;8:51-57.

37. Krieger DW, Demchuk AM, Kasner SE, Jauss M, Hantson L. Early clinical and radiological predictors of fatal brain swelling in ischemic stroke. Stroke 1999;30:287-292.

38. McCourt R, Gould B, Gioia L, Kate M, Coutts SB, Dowlatshahi D, et al. Cerebral perfusion and blood pressure do not affect perihematoma edema growth in acute intracerebral hemorrhage. Stroke 2014; 45:1292-1298.

39. Yong M, Kaste M. Association of characteristics of blood pressure profiles and stroke outcomes in the ECASS-II trial. Stroke 2008;39: 366-372.

40. Ohwaki K, Yano E, Nagashima H, Hirata M, Nakagomi T, Tamura A. Blood pressure management in acute intracerebral hemorrhage: relationship between elevated blood pressure and hematoma enlargement. Stroke 2004;35:1364-1367.

41. Suri MF, Suarez JI, Rodrigue TC, Zaidat OO, Vazquez G, Wensel A, et al. Effect of treatment of elevated blood pressure on neurological deterioration in patients with acute intracerebral hemorrhage. Neurocrit Care 2008;9:177-182. 